REVIEW

\title{
Tricks in dystonia: ordering the complexity
}

\author{
Vesper Fe Marie Llaneza Ramos, ${ }^{1}$ Barbara I Karp, ${ }^{2}$ Mark Hallett ${ }^{1}$
}

${ }^{1}$ Human Motor Control, National Institutes of Health, Bethesda, Maryland, USA ${ }^{2}$ National Institutes of Health, Bethesda, Maryland, USA

\section{Correspondence to}

Dr Mark Hallett, NIH, NINDS, Building 10, Room 7D 37,

10 Center Drive, Bethesda, MD 20814, USA; hallettm@ ninds.nih.gov

Received 16 October 2013 Revised 12 December 2013 Accepted 7 January 2014 Published Online First 31 January 2014

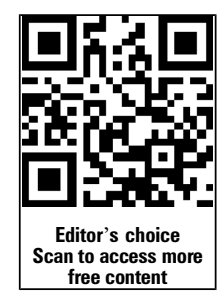

CrossMark

To cite: Ramos VFML, Karp BI, Hallett M. J Neurol Neurosurg Psychiatry 2014;85:987-993.

\section{ABSTRACT}

Sensory tricks are various manoeuvres that can ameliorate dystonia. Common characteristics are well known, but their variety is wide, sensory stimulation is not necessarily the critical feature, and their physiology is unknown. To enumerate the various forms of sensory tricks and describe their nature, research findings and theories that may elucidate their neurophysiologic mechanism, we reviewed the literature pertaining to sensory tricks, including variants like motor tricks, imaginary tricks, forcible tricks and reverse sensory tricks. On the basis of this information, we propose a new classification of sensory tricks to include its variants. We highlight neurophysiologic evidence suggesting that sensory tricks work by decreasing abnormal facilitation. We tie this with established dystonia pathogenesis and postulate that sensory tricks decrease abnormally increased facilitation to inhibition ratios in the dystonic brain. It appears worthwhile for patients to search for possible sensory tricks.

\section{INTRODUCTION}

Sensory tricks, the common English name for manoeuvres that temporarily improve dystonic posture, are a classic hallmark of primary dystonia and some cases of secondary dystonia. ${ }^{1}$ Also called 'geste antagoniste', from the description of the phenomenon in cervical dystonia by Henry Meige and Eugene Feindel in $1902,{ }^{2}$ contrary to the terminology, the movement of a trick does not always need to be antagonistic or in the opposing direction of the dystonia. ${ }^{3}$ The term 'antagonistic' might be better thought of in terms of a trick reversing the dystonia. Patel et al recently introduced the term, 'alleviating manoeuvre'. 4

We define 'sensory trick' as an episodic and specific manoeuvre that ameliorates dystonia in a manner that is not easily physiologically perceived as necessary to counteract the involuntary movement. Sensory input is not always necessary or sufficient. Motor tricks are defined as those sensory tricks involving voluntary movement as the apparent critical feature. ${ }^{5}$ The distinction between motor and sensory may be academic given the critical inter-relationship of motor and sensory function including sensory feedback from any movement. ${ }^{6}$ Originally, according to Meige and Feindel 'the mere threat of the gesture suffices...before the patient has actually touched his face....' ${ }^{2}$ One can question if the trick is the motor manoeuvre or the cognitive imagery of the performance of the manoeuvre. Indeed, imaginary tricks have been described, which are sensory tricks with mental imagery as the effective agent.
Atypical tricks include forcible tricks, which are manoeuvres similar to sensory tricks but necessitate the use of force, and are always antagonistic to the direction of the dystonia. These two characteristics differentiate forcible tricks from the other tricks previously described, leading one to question if they are indeed a sensory trick variant or simply the use of a force to antagonise the abnormal dystonic movement. We argue that forcible tricks result from force that is usually not as strong as necessary to actually counteract the dystonic contractions. Forcible tricks tend to provide longer relief and appear to be effective for more severe dystonia than classic sensory tricks. ${ }^{7}$ To add to the complexity of the topic are reverse sensory tricks, that is, sensory tricks that worsen dystonia, unlike all other tricks described previously, which ameliorate dystonia.

Being almost exclusive to dystonia, sensory tricks aid diagnosis. Though responses to sensory tricks have been described in exceptional cases of other movement disorders such as hemichorea-hemiballism, Parkinson tremor, ${ }^{8}$ and even psychogenic movement disorders, ${ }^{9}$ the recognition of typical features of sensory tricks is an important tool in dystonia diagnosis. Dystonic tremor may in some circumstances be differentiated from essential tremor by electromyography demonstration of cessation of tremor during sensory tricks for the former but not the latter. ${ }^{10}$ Wissel et al likewise reported a decrease in dystonic tremor amplitude in 10 of 13 patients with sensory tricks. $^{11}$

In this review article, to better understand the nature of sensory tricks and enumerate its various forms, we integrate literature related to sensory tricks from original experiments and expert opinions. Table 1 lists the variety of sensory tricks described in literature.

\section{THE NATURE OF A SENSORY TRICK}

Sensory tricks involve a stimulus with resultant change in dystonic muscle contraction. It is unclear what processing links these two events. Typical stimuli are light touches to a particular skin area that are noted to be sufficient even when the force applied is weak and ordinarily could not counteract dystonic contractions simply by overpowering them. ${ }^{3}$ Usually simple activities, ${ }^{7}$ some tricks can be complex and bizarre (table 1). They usually confer a temporary change in muscle activity and relief of dystonia, but there have been rare exceptions where the response is longer. ${ }^{3} 36$

Sensory tricks vary from person to person and within the same individual. The multiple and heterogenous features were misinterpreted as indicative of a psychogenic aetiology. 9 Another important 
Table 1 List of sensory tricks according to type of dystonia

\begin{tabular}{|c|c|}
\hline Dystonia & Sensory trick \\
\hline Cervical dystonia & 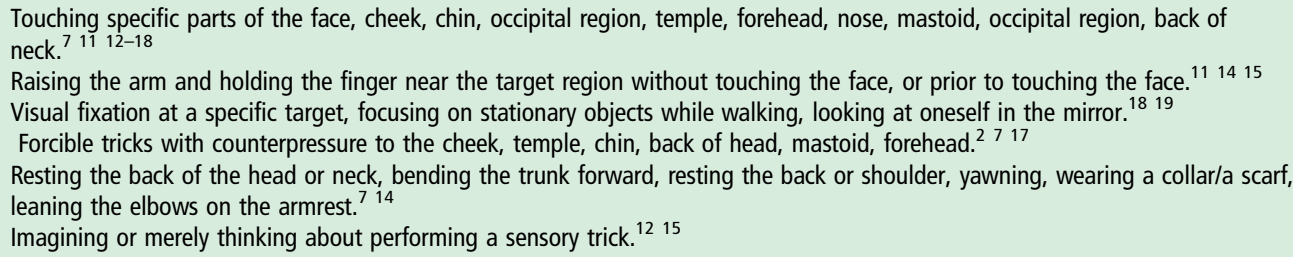 \\
\hline $\begin{array}{l}\text { Apraxia of eyelid opening, } \\
\text { blepharospasm }\end{array}$ & $\begin{array}{l}\text { Tight goggles or spectacles, Lundie Loops. }{ }^{17} 2021 \\
\text { Device inserted in glasses to mimic touch to lateral eyelid. }{ }^{22} \\
\text { Touching/pulling eyelids, tape on eyebrow. }{ }^{23} 24 \\
\text { Touching specific parts of the face (forehead, nose, side of eyelids, chin). }{ }^{17} 2224-26 \\
\text { Pushing back of the head. }{ }^{17} \\
\text { Massaging cheek bones, eyelid, forehead. }{ }^{17} \\
\text { Closing the jaw, chewing gum. }{ }^{12} 17 \\
\text { Touching bitemporal skin beside eyes. }^{27} \\
\text { Covering the eye. } \\
\text { Picking teeth. } \\
\text { Wearing a cap or turban. }\end{array}$ \\
\hline Meige syndrome & $\begin{array}{l}\text { Sleeping/relaxing, talking, singing/humming, pulling on upper eyelid, pinching back of the neck, yawning, belching, sucking in or } \\
\text { blowing out cheeks, drinking cold and/or alcoholic beverage. }{ }^{12} 28\end{array}$ \\
\hline $\begin{array}{l}\text { Lower cranial dystonia, oromandibular } \\
\text { dystonia }\end{array}$ & $\begin{array}{l}\text { Toothpick in mouth, holding object clenched between the teeth, dental splint, touching lip, touching lower corner of face. } \\
\text { 'Mandibular sensory trick device', } 29 \\
\text { Touching tongue to palate, biting lips, swallowing, pulling face up, bending neck forward. }{ }^{30} 31 \\
\text { Chewing gum, sucking. }{ }^{30-33} \\
\text { Kissing, whistling. } \\
\text { Pen/cigarette/tongue depressor in mouth. }{ }^{33} 34 \\
\text { Biting food/plastic between left upper and lower molars } \rightarrow \text { dental prosthesis device } 3 \mathrm{~mm} \text { above molar. }{ }^{3} \\
\text { Smiling, singing, talking, thinking about talking. } \\
\text { Biting piece of cotton or plastic. } \\
\text { Dental splint. } \\
\text { Playing with larger mouthpieces. }{ }^{35}{ }^{36} \\
5 \text { min ice massage of facial muscles. }\end{array}$ \\
\hline Laryngeal dystonia & Grimacing, laughing, loud background noise. ${ }^{38}$ \\
\hline Writer's cramp & $\begin{array}{l}5 \text { min immersion in cold water. } \\
\text { Shifting pen holding, holding pen between index finger and thumb vertically, writing with a closed fist. } \\
\text { Use pens of different sizes and calibres, using chalk and blackboard or painting. }{ }^{40} \\
\text { Touching specific part of the contralateral normal hand to a specific part of the dystonic hand. }{ }^{41}{ }^{42}\end{array}$ \\
\hline Runner's dystonia & $\begin{array}{l}\text { Holding hands over head, run in a clockwise direction, mental imagery of running in a clockwise direction. }{ }^{43} \\
\text { Beach walking, applying pressure with hand at hip. }\end{array}$ \\
\hline Camptocormia & Low-slung backpack, using wheeled walker, pressing back against hallway. ${ }^{44}$ \\
\hline DYT1 dystonia & Piano playing. ${ }^{45}$ \\
\hline
\end{tabular}

property is topography. There appears to be a specific body area, a catchment area, unique to each individual upon which stimuli is applied or thought of as applied, that leads to the experience of sensory tricks (figure 1). This topographic area does not always include the nerve territory distribution with regards to the dystonia or other sensory tricks in the same person.

Ochudlo et al called sensory tricks as a 'self-developed' but 'subconsciously-acquired' way of coping with dystonia and noted that sensory tricks tend to appear early in the course of the disease. ${ }^{7}$ Of nineteen patients with idiopathic lower cranial dystonia, ten patients discovered sensory tricks on their own and eight were discovered on initial clinical evaluation. ${ }^{29}$ Thus, patients may not be aware of a possible sensory trick. Among 50 patients with cervical dystonia and an effective sensory trick, the majority could not describe how they discovered their sensory trick. ${ }^{14}$ Patients often cannot recall the actual onset of sensory trick discovery. ${ }^{7}$

\section{Effectiveness}

Most sensory tricks are readily demonstrable. Martino et al documented that more than $90 \%$ of their patients reporting sensory tricks were able to demonstrate it to examiners. ${ }^{17}$ Reported prevalence for sensory tricks is about 17-89\%, with

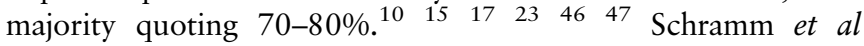
studied 26 cervical dystonia patients, $73 \%$ of whom had an effective sensory trick. They found a trend among those with effective sensory tricks to have head rotation to the left, shorter disease duration and larger maximal head rotation angle compared to patients without an effective sensory trick. ${ }^{15}$ Sensory tricks were less effective when the starting position of the head was directed towards the dystonic direction and work better when the starting head position was neutral or overcorrected. Even their comparison group of seven cervical dystonia patients without perceived effective sensory tricks had a significant decrease in electromyogram dystonic activity simply by directing their head position opposite to the dystonic activity. They concluded that the effectiveness of sensory tricks is strongly dependent on the starting head rotation angle. Deuschl et al showed that among 100 cervical dystonia patients, only 24\% had complete cessation of dystonic activity, 56\% had some decrease in dystonic activity, and $26 \%$ had no decrease with a change in head positioning towards the dystonia. ${ }^{10}$ We argue that this act of redirecting the head turning may, in itself, be a 


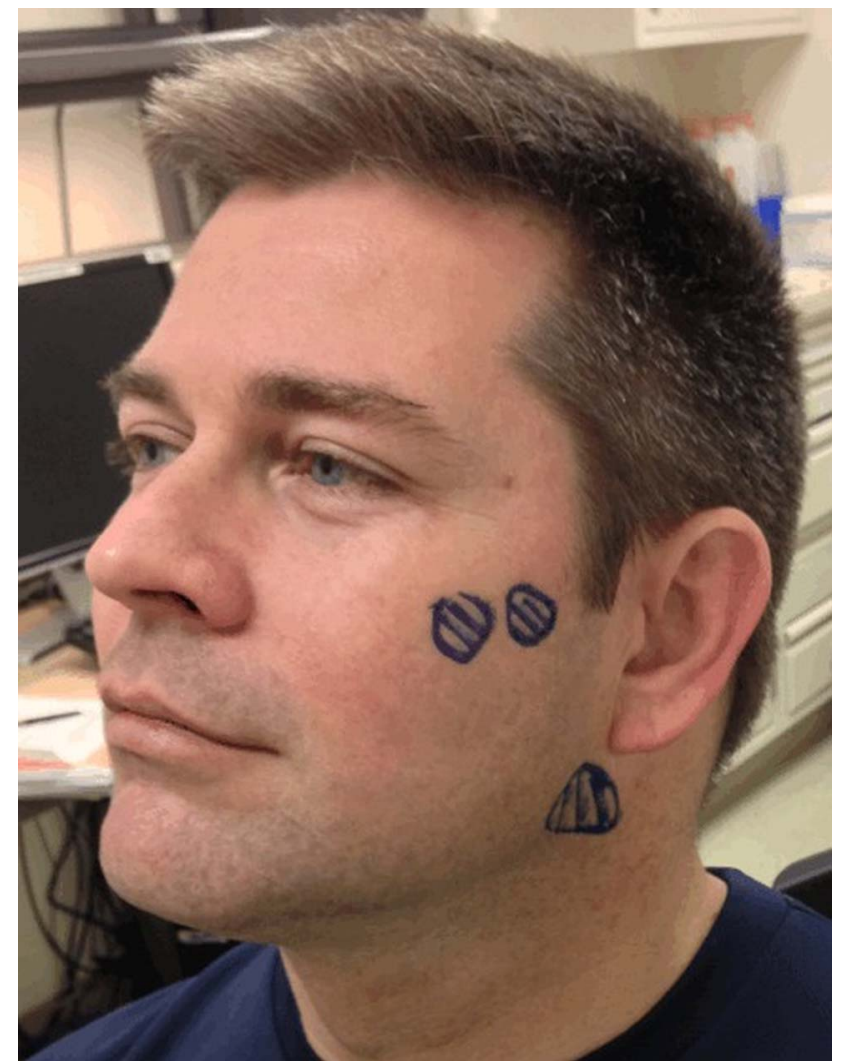

Figure 1 Topographic non-dermatomal areas of successful tactile stimuli for relief of cervical dystonia. A patient with cervical dystonia demonstrated effective sensory trick of touching areas shaded with marker.

sensory trick, or perhaps more aptly called a motor trick or forcible trick.

The duration of effect of sensory tricks upon introduction of the stimulus varies. Among 50 patients with cervical dystonia and effective sensory tricks, $29(58 \%)$ had a sensory trick response that lasted for more than $1 \mathrm{~min}$, while $42 \%$ had sensory trick responses that lasted from a few seconds to less than 1 min. ${ }^{14}$ Patients who had sensory tricks effective for more than $1 \mathrm{~min}$ tended to have shorter disease duration. Ochudlo et al described that most of their cervical dystonia patients with sensory tricks had effects lasting a few seconds, with only 2/33 patients having effects lasting from a few minutes to hours. ${ }^{7}$ There was no mention whether the trick stimulus had to be maintained to achieve the prolonged effect, but usually that is the case.

Sensory tricks tend to lose effectiveness over the course of the dystonia, even to the point of disappearing completely. In the Martino et al group of cervical dystonia and blepharospasm patients, 9.5\% had sensory tricks that disappeared. ${ }^{17}$ Muller et al reported a similar number of $5 / 50(10 \%)$ cervical dystonia patients losing sensory trick efficacy. ${ }^{14}$ Citing the association they found between shorter disease duration and longer duration of effectiveness of sensory tricks, the authors theorised that the perceived loss of sensory trick effectiveness may, in fact, be progression of the dystonia. Others noted that sensory tricks tend to lose effectiveness with increased disease severity. ${ }^{7} 48$

There is a trend towards an increased number of sensory tricks in patients with younger age of dystonia onset and increasing complexity of cervical dystonia. Age at the time of study, gender, disease duration and symptom severity had no significant effect on the presence of effective sensory tricks. ${ }^{15} 17$ Martino et al, however, did not show their data on disease duration and severity, and despite the trend, age of onset was found to be non-significant as well. They rated dystonia severity according to the Burke-Fahn-Marsden scale, and defined complexity as head deviation of more than $15^{\circ}$ in at least two planes. ${ }^{17}$ Schramm et al used Toronto Western Spasmodic Torticollis Rating Scale (TWSTRS) to measure severity and complexity. ${ }^{15}$ Deuschl et al used the clinical rating scale of Tsui and Calne, and found that effective sensory tricks were not associated with dystonia severity or duration. ${ }^{10}$ They noted that sensory tricks appeared to be more effective in patients with rotatory torticollis (61 of $72(85 \%)$ ) compared to other forms of torticollis. Kagi et al showed that shorter disease duration and lesser disease severity by TWSTRS score was associated with more effective sensory tricks. ${ }^{48}$ Aging affects brain plasticity, with younger patients generally having more plastic brains. It is unknown if the increased plasticity leads to more sensory tricks as a compensatory mechanism or as the starting point of the disease.

Sensory trick effectiveness can be judged with different criteria: by change in the dystonic position, using various clinical scales of dystonia severity, decrease in dystonic electromyogram activity, or the patient's subjective report of relief. With the goal of the patient's well-being, the last criteria may be most important. There are no reports in the literature of previously known sensory tricks worsening dystonia, or converting to reverse sensory tricks.

\section{Heterogeneity}

An impressive variety of sensory tricks have been described in literature and clinical anecdotes (table 1). Among 50 patients with cervical dystonia and effective sensory tricks, 27 (54\%) had more than one trick with a range of $2-5 .{ }^{14}$ Sensory tricks are often multisensory, challenging the notion of what is a necessary stimulus.

\section{Tactile and proprioceptive stimuli}

Physical stimulation by the patient himself is the most common trick, and usually the most effective, but it is not always necessary. ${ }^{15}$ Proprioception appears to be a stronger stimulus for eliciting sensory tricks than tactile stimuli in cervical dystonia. Muller et al's review of cervical dystonia patients with effective sensory tricks also showed that $10 / 50(20 \%)$ were able to maintain continuous efficacy of the sensory tricks as long as the body position eliciting the sensory tricks was maintained. ${ }^{14}$ This preference for the neutral or overcorrected position appears counterintuitive given that sensory tricks need to work when the dystonic posture is present, and not in the normal or overcorrected phase. Perhaps it is really the case that sensory tricks work best in the prevention mode, rather than correction mode. A tactile touch to the cheek was rendered more effective as a trick by moving the starting head position away from the dystonic direction. ${ }^{15}$ With the sensory trick touch performed by the investigator, instead of the patient, there was a lesser, but still significant, reduction in electromyogram activity. Replacing the touch of a finger with a plastic stick, mere imagining of the sensory trick, and an performing the first part of the trick task by raising the arm but not physically touching the finger to the cheek appeared less, but still significantly, effective as measured by decreased dystonic activity in electromyogram. ${ }^{15}$

Effective sensory tricks in blepharospasm are predominantly tactile, including eyelid pressure, rubbing, or covering the eye; some patients even benefit from adding a device to their 
spectacles that places continuous pressure on the lateral aspect of eyelids. ${ }^{22}$ Wearing goggles was hypothesised to result in a proprioceptive input that helped relieve symptoms. However, continuous use diminished effectiveness. ${ }^{20}$ For oromandibular dystonia, sensory tricks usually involve clenching an object, such as a toothpick in between the teeth, or a light touch to a part of the face. ${ }^{29}$ Dental splinting was reported successful. ${ }^{35} 36$

\section{Visual and auditory stimuli}

Cervical dystonia improved for $90 \mathrm{~s}$ with visual sensory tricks, such as mirror viewing, staring at a specific object or visual fixation, only to recur again after $10 \mathrm{~s}$ from eye closure or eye movement. ${ }^{18}$ This reflects the temporally transient and inputdependent characteristics of sensory tricks. A theory to explain this, supported by positron emission tomography studies, is that visual information affects cervical dystonia by providing a compensatory mechanism for faulty proprioception by enhancing pathways between the occipital and parietal lobes. ${ }^{18}$

Stojanovic et al described a patient with spasmodic adductor laryngeal dystonia relieved by loud background noise, such that the patient opted to turn the radio on when speaking to people. ${ }^{38}$ Fiberoptic laryngoscopy confirmed the change in dystonic laryngeal muscle activity with this trick. The authors theorised that auditory input provided feedback to induce the patient to speak more clearly, as in normal people producing louder vocalisations when placed in a noisy environment. Piano playing led to significant improvement of all dystonic muscles, evidenced by electromyogram, in a single patient with DYT1 dystonia. $^{45}$ The degree of improvement decreased when the sound of the piano was turned off indicating that this was not a motor trick. The report did not, however, evaluate dystonic activity recorded with just listening to piano music, without playing, which would have been helpful in demonstrating an auditory sensory trick. There are also reports on auditory stimuli worsening dystonia that will be discussed below.

No gustatory or olfactory stimuli as sensory tricks eliciting dystonia improvement have been described in the literature.

\section{Thermal stimuli}

Dipping the hand in $15^{\circ}$ centigrade water for 5 min improved dystonic symptoms among 7/10 (70\%) patients with writer's cramp. ${ }^{39}$ Increased speed of writing lasted for about $25 \mathrm{~min}$. Warming induced worsening of symptoms for about $10 \mathrm{~min}$ in three patients. Kim et al used electromyography to demonstrate 1 min improvement of dystonia after a $5 \mathrm{~min}$ ice massage to affected muscles in a case of embouchure dystonia. ${ }^{37}$ Both papers suggested that cooling ameliorated dystonia similar to sensory tricks, which may be by way of decreasing muscle spindle or skin receptor activity.

\section{Imaginary tricks}

Some patients are able to induce a relief of dystonia by imagining the performance of their own sensory tricks, ${ }^{1} 1215$ or other mental imagery, such as picturing normal head posture. ${ }^{43} 4950$ Suzuki et al described a man with runner's dystonia involving neck and trunk flexion brought about by running counterclockwise. Asking the patient to imagine running clockwise improved dystonia. ${ }^{43}$ Imaginary tricks show that sensory input is not necessary in all circumstances, which raises the question of what critical features of imagination are shared with sensory input that induce the motor response and relief of dystonia. Imagination of movement leads to very similar patterns of brain activation as movement itself. $^{51} 52$
Motor tricks and the atypical tricks: forcible tricks and reverse sensory tricks

Sensory tricks often involve voluntary movement input. The mere act of almost touching the finger to the cheek was sufficient to effect a decrease in electromyographic activity in dystonic muscles in 13/25 (52\%) cervical dystonia patients with sensory tricks, while tactile contact using an examiner's finger failed to effect any electromyogram change in 18 (72\%). ${ }^{11}$ Schramm et al surmised that voluntary activation of antagonistic muscles was important for sensory tricks in jaw-opening dystonia. ${ }^{53}$ Whether this is a motor trick or forcible trick, or simply an opposing manoeuvre against the dystonic contraction is difficult to ascertain. It may be possible to differentiate between these options by the degree of complexity and amount of force and direction of the movement.

Reverse sensory tricks are sensory trick-like stimuli that result in worsening of dystonia. A cohort of 47 cervical dystonia patients revealed a prevalence of $12.8 \%$ for reverse sensory tricks. $^{54}$ These tricks produced increased electromyographic activity in the neck muscles corresponding to known cervical dystonia. One of the patients in the cohort was well described with a heterogenous set of reverse sensory tricks that worsened cervical dystonia, namely neck and back of the head tactile stimulation and eye closure. ${ }^{54}$ The nature of the described reverse sensory tricks appeared similar in various aspects to sensory tricks, such as topographic specificity and influence of posture, significantly affecting dystonic symptoms in a manner that is not easily explainable by physiology. The existence of reverse sensory tricks emphasises the unique individual nature of sensory tricks. Stimuli or acts that constitute sensory tricks in one patient may be reverse sensory tricks in another.

Tempel and Perlmutter demonstrated co-contraction of upper extremity flexors and extensors with the application of a vibration stimulus to a hand and lasting for the duration of the stimulus in 6/11 patients with focal dystonia. ${ }^{55}$ Similarly, Kaji et al induced dystonic movements in patients with writer's cramp significantly more easily than they were able to elicit wrist and finger flexion in healthy volunteers by applying vibration to the palm or tendons of forearm muscles. ${ }^{41}$

Motor tricks can also be reverse. Walking, writing, running, heavy lifting or even a supine position, may also lead to worsening cervical dystonia in some patients.

\section{NEUROPHYSIOLOGY}

Exactly how sensory tricks work is unclear. Physiological investigations with different methods provide some clues.

\section{Electromyography}

Electromyography is useful in the study of sensory tricks for objective analysis. Sensory tricks result in decreased electromyographic activity in dystonic muscles, which is the physiological correlate of the reduction in dystonia. Deuschl et al showed complete cessation of dystonic electromyographic activity in the sternocleidomastoid, splenius and trapezius muscles with sensory tricks of touching the chin with the hand. ${ }^{10}$ Electromyographic changes with sensory tricks include a reduction of recruitment density and amplitude, and also a possible increase in tonic muscle activation in those muscles with phasic electromyography at rest. ${ }^{11} 14$ The increase in tonic activity was postulated to serve to counteract dystonic movements and to produce harmonisation of movement. Wissel at al showed that tactile sensory tricks, such as stroking the face with the hand, can have a temporal profile of three phases: (a) Movement phase I-from the initiation of the 
trick to just prior to tactile contact; (b) Contact and posture phase-from start to end of tactile contact; and (c) Movement phase II-from the removal of tactile contact to the end of the trick movement. ${ }^{11}$ Their results revealed a reduction of electromyographic activity even in Movement phase I in 13/25 (52\%) patients, and in Movement phase II in six (24\%) patients. ${ }^{11}$ ) This study clearly demonstrated that tactile contact is not necessary for sensory tricks in all patients.

Schramm et al studied seven patients with jaw-opening dystonia and sensory(motor) tricks of placing a stick between cheek and teeth, biting down with a stick, and biting without a stick. ${ }^{53}$ They found that some sensory tricks failed to improve patient's subjective report of dystonic condition, even though sensory tricks correlated with decreased activity on surface electromyography, as well as experimenter-rating scores of articulation.

As mentioned, Kaji et al demonstrated with electromyography that dystonia could be induced by vibration. ${ }^{41}$ Additionally, the induced movements were prevented with lidocaine injections into affected muscles, suggesting that spindle-afferent traffic provoked the dystonia. Thus, this phenomenon is an exaggeration of the tonic vibration reflex and would be one mechanism for a reverse sensory trick. Oppositely, as noted earlier, dampening of spindle-afferent traffic might be a mechanism for some sensory tricks.

\section{Blink reflex studies}

Gomez-Wong et al studied prepulse inhibition of the blink reflex in 17 patients with blepharospasm, eight (47\%) of whom had effective sensory tricks, and in 11 healthy volunteers. ${ }^{25}$ Patients with sensory tricks had R2 inhibition similar to healthy volunteers, while patients without sensory tricks had significantly decreased R2 inhibition. That is, patients with sensory tricks had a normal prepulse inhibition. The authors theorised that sensory tricks act in a similar manner as a prepulse stimulus, leading to inhibition of dystonic overcontraction.

In another publication, Gomez-Wong et al described their findings in eight patients with blepharospasm, and effective sensory tricks of touching the facial region near the orbit, and in six healthy volunteers mimicking sensory tricks using single and paired stimuli blink reflex studies. ${ }^{24}$ The R2 response was higher at baseline in patients with blepharospasm compared to healthy volunteers. After sensory tricks were applied, the R2 was decreased in all subjects even though the blepharospasm patients had a smaller reduction compared to volunteers. There were no changes in the blink reflex excitability curve. The authors hypothesised that sensory tricks improve blepharospasm transiently by a decrease in the gain of trigeminofacial reflexes. That is, sensory tricks induce a decrease in the magnitude of the reflex response to exteroceptive stimuli.

\section{Transcranial magnetic stimulation}

Tesfaghebriel et al studied sensory tricks in five patients with cervical dystonia and in seven healthy volunteers using pairedpulse transcranial magnetic stimulation. ${ }^{56}$ They found that short intracortical inhibition in the hand was unchanged, while intracortical facilitation was significantly increased in cervical dystonia patients versus healthy volunteers, and that the use of sensory tricks decreased the abnormal intracortical facilitation in cervical dystonia patients. This observation parallels the results of the previously discussed blink reflex study by Gomez-Wong et $a l^{24}$ That is, sensory tricks did not alter inhibition directly (demonstrated by the absence of changes in short intracortical inhibition or blink reflex recovery curves) but decreased abnormally enhanced facilitation (decreased intracortical facilitation and decreased
R2 blink reflex). Similar observation using two different physiologic methods in different types of dystonia suggests a common mechanism of response (figure 2).

\section{Imaging studies}

A positron emission tomography and electromyogram study of seven cases of sensory trick-responsive cervical dystonia revealed that sensory tricks increased parietal and bilateral visual cortex activation, with concomitant deactivation of the contralateral supplementary motor area and primary sensorimotor cortices. ${ }^{13}$ The authors theorise that sensory tricks result in a perceptual change, as evidenced by the parietal cortex activation, which acts as an 'arousal stimulus' for correction to the normal nondystonic state. They further highlighted the activation of the visual cortices bilaterally even though subjects had their eyes closed, suggesting that such activity might be necessary to the physiology of sensory tricks. In another study, a tapping task performed by patients with writer's cramp demonstrated hypermetabolism in the left M1 and S1, unilateral anterior cingulate, supplementary motor area and cerebellum. ${ }^{57}$ Thus, Naumann et al's finding of hypometabolism in the supplementary motor area, ${ }^{13}$ an area shown to have facilitatory effects on the primary motor cortex ${ }^{58}$ and to be hyperactive in dystonia, ${ }^{57}$ is congruent with aforementioned neurophysiologic data that sensory tricks decrease abnormal cortical facilitation.

\section{MECHANISTIC HYPOTHESIS}

That effectiveness of sensory tricks was associated with improved cross-modal tactile/visual temporal discrimination, but not with unimodal tasks, imply that the process is complex. ${ }^{48}$ The complexity of inputs that can be effective suggests that the influence is in the premotor structures, such as the supplementary motor area. Thus, imagination can be effective and dystonia can be diminished even before the hand reaches the chin.

Gomez-Wong et al, based on prepulse inhibition studies, theorised that an abnormal 'gating mechanism' in dystonia results in reflex pathologic circuits, which can be normalised by sensory tricks. ${ }^{25}$ There is good evidence for the existence of abnormal gating in dystonia, including somatosensory-evoked potential studies ${ }^{59}$ that showed abnormalities for the P22 and N30 in patients with writer's cramp. The authors consider that sensory tricks provide another input to the dystonic brain to correct alterations in brain sensitivity to existing inputs. Kaji et al have theorised that dystonia is secondary to a sensory inputmotor output mismatch between a frequently used motor subroutine and a fixed sensory afferent. ${ }^{41}$ Similarly, Abbruzzese
A

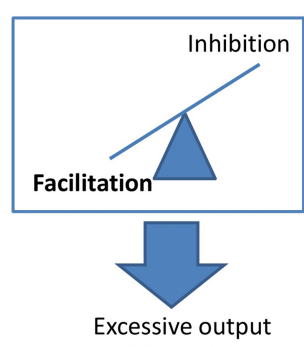

(dystonia)

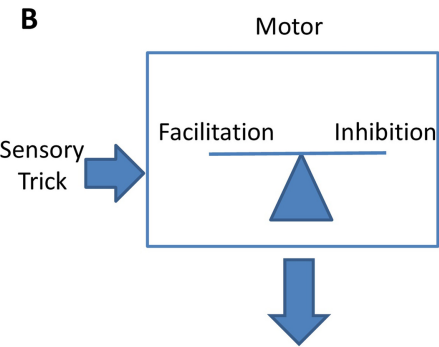

Normal output
Figure 2 Diagrammatic illustration of the possible mechanism of sensory tricks. (A) In patients with dystonia, there is an abnormally high facilitation to inhibition ratio leading to the excessive movement. (B) With the sensory input of a trick, the facilitation is reduced, normalising the ratio and improving motor output. 
and Berardelli proposed that sensory tricks act by an adjustment of abnormal link between sensory input and motor output. $^{60}$

Physiological studies suggest that sensory tricks lead to a 'normalisation' of the dystonic brain specifically by decreasing the abnormal cortical facilitation. ${ }^{24} 56$ Dystonia pathogenesis likely involves decreased inhibition at multiple levels of the nervous system. The mechanism is likely to be a balancing of the ratio of inhibition to facilitation. That is, in dystonia, there is an increased facilitation to inhibition ratio. We hypothesise that sensory tricks decrease this ratio, thus ameliorating dystonia (figure 2).

Patients with dystonia have a loss of surround inhibition, decreasing selective control of movement, leading to overflow and perhaps to dystonic movements. If sensory tricks reduce the facilitation to inhibition ratio of the field surrounding the moving muscle, and, if the dystonic muscle is in this surround, the dystonia will be suppressed. For example, eyelids are in the surround cerebral territory for muscles involved in speaking, and speaking will often improve blepharospasm. More direct evidence would be needed to support this hypothesis.

\section{SHOULD WE INDUCE A SENSORY TRICK?}

Given the benefit of sensory tricks, and the key features of selfdiscovery, self-application and possible need for attention, it may be worth having patients systematically try to identify and elicit sensory tricks. Biofeedback, such as electromyography, might be used to identify sensory tricks and perhaps even to facilitate their development. Attention to a possible trick might enhance the response.

Citing sensory tricks as low-cost, risk-free alternative treatments with potentially dramatic efficacy, Frucht et al called to seek out sensory tricks in all patients with oromandibular dystonia. ${ }^{3}$ They may be especially important to explore in specific types of dystonia, such as jaw-opening dystonia, for which botulinum toxin is not very effective. ${ }^{53}$ Avoiding invasive and uncomfortable injections, especially in delicate areas such as eyelids, might make patients more willing to explore sensory tricks.

The transitory nature of benefit from sensory tricks is a problem. Careful analysis of sensory tricks may lead to the fabrication of effective mimicry devices, that allow for more practical usage for the treatment of specific dystonias, as in dental implants in oromandibular dystonia, ${ }^{36} 53$ or use of goggles in blepharospasm. ${ }^{172022}$ Lo et al fabricated dental splints in eight patients with idiopathic lower cranial dystonia, with each device specific for each patient's known sensory tricks. Six out of eight had immediate improvement of dystonic symptoms, but only three patients enjoyed a response sustained for longer than a few weeks. ${ }^{29}$

We also need to look more deeply into the negative aspects of sensory tricks. Their transitory nature may result in frustration among patients, who suspect that normal function lies beneath the dystonia. ${ }^{8}$ Inconsistency of results is a further concern, such as in a patient with blepharospasm with sensory tricks that fail while driving.

If possible, might deliberately inducing sensory tricks be valuable or worsen dystonia? Repetitive use of muscles likely leads to dystonia. ${ }^{6}$ Several studies have not found any association between sensory tricks and disease duration or severity, ${ }^{10} 17$ arguing against the relation of sensory tricks to disease progression.

\section{CONCLUSION}

Sensory tricks are a fascinating feature of the mysterious motor disturbances in dystonia.

This review points out variants, such as motor tricks, imaginary tricks, forcible tricks and reverse sensory tricks. Our research points towards recommending to patients to search for them. More studies are needed to elucidate the physiology of sensory tricks, but a modulation of premotor sensorimotor integration, normalising the facilitation to inhibition ratio may be relevant.

Acknowledgements The authors wish to thank Dr Codrin Lungu, Dr Angela Holmes and Dr Ajay Pillai for their valuable assistance with the preparation of this manuscript.

Contributors VFMLR wrote the original draft and BIK and MH revised and edited it.

Funding This work was supported by the National Institutes of Health and the National Institute of Neurological Disorders and Stroke. The views expressed in written materials or publications do not necessarily reflect the official policies of the Department of Health and Human Services; nor does mention by trade names, commercial practices, or organisations imply endorsement by the US Government. VR was supported by the Dystonia Medical Research Foundation.

Competing interests None.

Provenance and peer review Commissioned; externally peer reviewed.

\section{REFERENCES}

1 LeDoux MS. Dystonia: phenomenology. Parkinsonism Relat Disord 2012;18(Suppl 1):S162-4.

2 Poisson A, Krack P, Thobois $S$, et al. History of the 'geste antagoniste' sign in cervical dystonia. J Neurol 2012;259:1580-4.

3 Frucht $\mathrm{S}$, Fahn $\mathrm{S}$, Ford $\mathrm{B}$, et al. A geste antagoniste device to treat jaw-closing dystonia. Mov Disord 1999;14:883-6.

4 Patel N, Jankovic J, Hallett M. Sensory aspects in movement disorders. Lancet Neurol 2014;13:100-12.

5 McClinton S, Heiderscheit BC. Diagnosis of primary task-specific lower extremity dystonia in a runner. J Orthop Sports Phys Ther 2012;42:688-97.

6 Hallett M. Is dystonia a sensory disorder? Ann Neurol 1995;38:139-40.

7 Ochudlo S, Drzyzga K, Drzyzga LR, et al. Various patterns of gestes antagonistes in cervical dystonia. Parkinsonism Relat Disord 2007;13:417-20.

8 Lewitt PA, Gostkowski MT. "Sensory Trick" in Hemichorea-Hemiballism and in Parkinson's Disease Tremor. Mov Disord 2010;25:1312-13.

9 Munhoz RP, Lang AE. Gestes antagonistes in psychogenic dystonia. Mov Disord 2004;19:331-2.

10 Deuschl G, Heinen F, Kleedorfer B, et al. Clinical and Polymyographic Investigation of Spasmodic Torticollis. J Neurol 1992;239:9-15.

11 Wissel J, Muller J, Ebersbach G, et al. Trick maneuvers in cervical dystonia: investigation of movement- and touch-related changes in polymyographic activity. Mov Disord 1999:14:994-9.

12 Greene PE, Bressman S. Exteroceptive and interoceptive stimuli in dystonia. Mov Disord 1998;13:549-51.

13 Naumann M, Magyar-Lehmann S, Reiners K, et al. Sensory tricks in cervical dystonia: perceptual dysbalance of parietal cortex modulates frontal motor programming. Ann Neurol 2000;47:322-8.

14 Muller J, Wissel J, Masuhr F, et al. Clinical characteristics of the geste antagoniste in cervical dystonia. J Neurol 2001;248:478-82.

15 Schramm A, Reiners K, Naumann M. Complex mechanisms of sensory tricks in cervical dystonia. Mov Disord 2004;19:452-8.

16 Tang JKH, Mahant N, Cunic D, et al. Changes in cortical and pallidal oscillatory activity during the execution of a sensory trick in patients with cervical dystonia. Exp Neurol 2007:204:845-8.

17 Martino D, Liuzzi D, Macerollo A, et al. The phenomenology of the geste antagoniste in primary blepharospasm and cervical dystonia. Mov Disord 2010;25:407-12

18 Lee CN, Eun MY, Kwon DY, et al. "Visual sensory trick" in patient with cervical dystonia. Neurol Sci 2012;33:665-7.

19 Boyd JT, Fries TJ. Novel expression of a visual geste antagoniste in cervical dystonia. Mov Disord 2005;20:S31-S.

20 Hirayama M, Kumano T, Aita T, et al. Improvement of apraxia of eyelid opening by wearing goggles. Lancet 2000;356:1413.

21 Ramasamy B, Rowe F, Freeman G, et al. Modified Lundie loops improve apraxia of eyelid opening. J Neuroophthalmol 2007:27:32-5.

22 Bain PG, Liu X, Aziz TZ. Increase in the tactile catchment area of a sensory trick for alleviating blepharospasm following pallidal DBS. Mov Disord 2009;24:624-6. 
23 Grandas F, Elston J, Quinn N, et al. Blepharospasm: a review of 264 patients. J Neurol Neurosurg Psychiatry 1988;51:767-72.

24 Gomez-Wong E, Marti MJ, Cossu G, et al. The 'geste antagonistique' induces transient modulation of the blink reflex in human patients with blepharospasm. Neurosci Lett 1998:251:125-8.

25 Gomez-Wong E, Marti MJ, Tolosa E, et al. Sensory modulation of the blink reflex in patients with blepharospasm. Arch Neurol 1998;55:1233-7.

26 Ragothaman M, Govindappa ST, Muthane UB. The "Indian turban trick": a novel sensory trick in blepharospasm. Mov Disord 2007;22:1516.

27 Krack P, Marion MH. "Apraxia of lid opening," a focal eyelid dystonia: clinical study of 32 patients. Mov Disord 1994;9:610-15.

28 Jankovic J, Ford J. Blepharospasm and orofacial-cervical dystonia: clinical and pharmacological findings in 100 patients. Ann Neurol 1983;13:402-11.

29 Lo SE, Gelb M, Frucht SJ. Geste antagonistes in idiopathic lower cranial dystonia. Mov Disord 2007;22:1012-17.

30 Sankhla C, Lai EC, Jankovic J. Peripherally induced oromandibular dystonia. J Neurol Neurosurg Psychiatry 1998;65:722-8.

31 Baik JS, Park JH, Kim JY. Primary lingual dystonia induced by speaking. Mov Disord 2004:19:1251-2.

32 Tan EK, Chan LL. Sensory tricks and treatment in primary lingual dystonia. Mov Disord 2005:20:388.

33 Kang SY, Kim H, Ma HI, et al. Highly task-specific oromandibular dystonia in a telephone operator. Eur J Neurol 2011;18:E136-E.

34 Kleopa KA, Kyriakides T. A novel movement disorder of the lower lip. Mov Disord 2004;19:663-6.

35 Frucht SJ, Fahn S, Greene PE, et al. The natural history of embouchure dystonia. Mov Disord 2001;16:899-906.

36 Satoh M, Narita M, Tomimoto H. Three Cases of Focal Embouchure Dystonia: Classifications and Successful Therapy Using a Dental Splint. Eur Neurol 2011:66:85-90.

$37 \mathrm{Kim}$ JS, An JY, Lee KS, et al. Cooling can relieve the difficulty of playing the tuba in a patient with embouchure dystonia. Mov Disord 2007;22:2291-2.

38 Stojanovic M, Kostic V, Stankovic $\mathrm{P}$, et al. Improvement in laryngeal dystonia with background noise. Mov Disord 1997;12:249-50.

39 Pohl C, Happe J, Klockgether T. Cooling improves the writing performance of patients with writer's cramp. Mov Disord 2002;17:1341-4.

40 Marsden CD, Sheehy MP. Writer's cramp. Trends Neurosci 1990;13:148-53.

41 Kaji $\mathrm{R}$, Rothwell JC, Katayama $\mathrm{M}$, et al. Tonic vibration reflex and muscle afferent block in writer's cramp. Ann Neurol 1995;38:155-62.
42 Hauser RA, Burke DA. Demonstration of a sensory trick in a case of writer's cramp. Mov Disord 2002;17:427.

43 Suzuki K, Izawa N, Aiba $\mathrm{S}$, et al. Interoceptive sensory trick for runner's dystonia. Mov Disord 2011;26:758-9.

44 Gerton BK, Theeler B, Samii A. Backpack treatment for camptocormia. Mov Disord 2010;25:247-8

45 Kojovic M, Parees I, Sadnicka A, et al. The brighter side of music in dystonia. Arch Neurol 2012:69:917-9.

46 Jahanshahi M. Factors that ameliorate or aggravate spasmodic torticollis. J Neurol Neurosurg Psychiatry 2000;68:227-9.

47 Martino D, Di Giorgio A, D'Ambrosio E, et al. Cortical Gray Matter Changes in Primary Blepharospasm: A Voxel-Based Morphometry Study. Mov Disord 2011;26:1907-12.

48 Kagi G, Katschnig P, Fiorio M, et al. Sensory tricks in primary cervical dystonia depend on visuotactile temporal discrimination. Mov Disord 2013;28:356-61.

49 Lo SE, Frucht SJ. Is focal task-specific dystonia limited to the hand and face? Mov Disord 2007:22:1009-11.

50 Torres-Russotto D, Perlmutter JS. Task-specific dystonias: a review. Ann N Y Acad Sci 2008;1142:179-99.

51 Hanakawa T, Dimyan MA, Hallett M. Motor planning, imagery, and execution in the distributed motor network: a time-course study with functional MRI. Cereb Cortex 2008;18:2775-88.

52 Hallett M, Iseki K. Real and imaginary gait. Mov Disord 2012;27:1473-4.

53 Schramm A, Classen J, Reiners K, et al. Characteristics of sensory trick-like manoeuvres in jaw-opening dystonia. Mov Disord 2007:22:430-3.

54 Asmus F, von Coelln R, Boertlein A, et al. Reverse Sensory Geste in Cervical Dystonia. Mov Disord 2009;24:297-300.

55 Tempel LW, Perlmutter JS. Abnormal vibration-induced cerebral blood flow responses in idiopathic dystonia. Brain 1990;113(Pt 3):691-707.

56 Tesfaghebriel HC, Amadio S, Del Carro U, et al. Effect of sensory tricks on brain excitability in cervical dystonia: TMS study. Clin Neurophysiol 2011;122:S85.

57 Lerner $A$, Shill $H$, Hanakawa $T$, et al. Regional cerebral blood flow correlates of the severity of writer's cramp symptoms. Neuroimage 2004;21:904-13.

58 Arai N, Lu MK, Ugawa Y, et al. Effective connectivity between human supplementary motor area and primary motor cortex: a paired-coil TMS study. Exp Brain Res 2012:220:79-87.

59 Murase $\mathrm{N}$, Kaji R, Shimazu $\mathrm{H}$, et al. Abnormal premovement gating of somatosensory input in writer's cramp. Brain 2000;123(Pt 9):1813-29.

60 Abbruzzese G, Berardelli A. Sensorimotor integration in movement disorders. Mov Disord 2003; 18:231-40. 